\title{
Narrow band imaging-assisted transurethral resection for non-muscle invasive bladder cancer significantly reduces residual tumour rate
}

\author{
Evelyne C. C. Cauberg • Charalampos Mamoulakis • \\ Jean J. M. C. H. de la Rosette • Theo M. de Reijke
}

Received: 21 October 2010/ Accepted: 4 February 2011/Published online: 25 February 2011

(c) The Author(s) 2011. This article is published with open access at Springerlink.com

\begin{abstract}
Purpose To investigate whether narrow band imaging (NBI)-assisted transurethral resection (TUR) (NBI-TUR) has an impact on non-muscle invasive bladder cancer (NMIBC) residual tumour rate compared to white light (WL)-assisted TUR (WL-TUR).

Methods Patients with NMIBC treated with either NBIor WL-TUR were compared in a frequency-matched indexcontrol setting. During NBI-TUR, all suspicious lesions identified by either WL or NBI were resected. Index patients (NBI-TUR, $n=40$ ) were prospectively recruited and control patients (WL-TUR, $n=120$ ) were retrospectively collected, whilst being blinded for their first followup (fFU)-status. Non-radical TUR cases, patients without evidence of urothelial carcinoma in the pathology specimen and those with isolated carcinoma in situ or muscle invasive disease were excluded. Matching was based on the risk of tumour recurrence defined by (a) the EORTC risk score for recurrence and (b) the administration or not of one single chemotherapeutic intravesical instillation immediately after TUR. All patients underwent routine follow-up with WL cystoscopy supplemented with cytology at 3 months or re-TUR in selected cases. The residual tumour rates at fFU (RR-fFU) of patients with NMIBC submitted to either NBI- or WL-TUR were compared.
\end{abstract}

E. C. C. Cauberg $(\bowtie) \cdot$ C. Mamoulakis .

J. J. M. C. H. de la Rosette - T. M. de Reijke

Department of Urology, Academic Medical Center,

P.O. box 22660, 1100 DD Amsterdam, The Netherlands

e-mail: e.c.cauberg@amc.nl

Present Address:

C. Mamoulakis

Department of Urology, University of Crete, Medical School,

University Hospital of Heraklion, Crete, Greece
Results Baseline patient and tumour characteristics were comparable between groups. The RR-fFU for WL- and NBI-TUR was $30.5 \%$ (36 out of 118 patients) and $15.0 \%$ (6 out of 40 patients), respectively (OR: 2.7 , one-sided $95 \%$ CI: $1.2-6.1 ; P=0.03)$.

Conclusion NBI-TUR decreases residual tumour rate significantly when compared to a matched cohort of WL-TUR.

Keywords Cystoscopy - Narrow band imaging · Recurrence $\cdot$ Transurethral resection - Urinary bladder neoplasms

\section{Introduction}

One of the major challenges in the management of nonmuscle invasive bladder cancer (NMIBC) is to reduce the high frequency of early recurrences, detected in up to $45 \%$ of the patients at first follow-up (fFU) cystoscopy, 3 months following transurethral resection (TUR) [1]. These recurrences may be "true" recurrences due to aggressive tumour biology or implantation of floating cancer cells. However, it is believed that most of these early "recurrences" in fact are overlooked or residual tumours, which stresses the importance of TUR quality [1]. Although a re-TUR contributes to a more complete resection [2-5], it is an additional invasive procedure, increasing the overall patient burden and healthcare costs. Therefore, instead of repeating the standard procedure, improving the technique itself represents a challenge.

Narrow band imaging (NBI) system (Olympus, Tokyo, Japan) is a diagnostic modality recently added to the urologist's armamentarium. It is an optical tool, independent of fluorescent agents, aiming at improving the 
visualisation of NMIBC by filtering WL into two narrow bandwidths of 415 and $540 \mathrm{~nm}$, which correspond to blue and green light, respectively. Due to the fact that these wavelengths are specifically absorbed by haemoglobin, the contrast between normal urothelium and vascular structures is enhanced. We have previously reported that NBI cystoscopy improves the detection of NMIBC compared to standard WL cystoscopy [6]. The aim of this study is to investigate whether this improved detection rate during NBI-assisted TUR (NBI-TUR) translates into a decrease in NMIBC residual tumour rate compared to WL-TUR.

\section{Materials and methods}

\section{Study design}

The residual tumour rate at fFU (RR-fFU) of patients with NMIBC submitted to either NBI- or WL-TUR in our centre was compared in a frequency-matched index-control setting. Index patients submitted to NBI-TUR were prospectively recruited. Control patients submitted to WL-TUR were retrospectively collected, whilst being blinded for their fFU status. Matching was based on the risk of tumour recurrence defined by (a) the European Organization for Research and Treatment of Cancer (EORTC) risk score for recurrence [7] stratifying patients into three distinct groups (low-, intermediate- and high risk) as proposed by the European Association of Urology (EAU) working group [8] and (b) the administration or not of one single chemotherapeutic intravesical instillation immediately after TUR. By combining these two criteria, six different matching subgroups were generated.

All patients underwent routine follow-up with flexible WL cystoscopy supplemented by bladder-wash out cytology at 3 months or re-TUR in selected cases. The indication for immediate and/or adjuvant instillations was based on risk group criteria and not imposed by the study protocol. Histopathological staging and grading was based on the TNM 2002 [9] and the WHO 1973 guidelines [10]. The prospective part of this study (NBI-TUR group) was approved by the local medical ethics committee.

Patient selection and procedures

From September 2007 to January 2010, consecutive patients with a suspicion of primary or recurrent bladder cancer based on cystoscopy or cytology findings were prospectively enrolled and submitted to NBI-TUR. The procedure has been previously described by Cauberg et al. [6]. In brief, under anaesthesia, all patients underwent WL cystoscopy followed by NBI cystoscopy. All tumours or suspicious areas were mapped and subsequently resected or biopsied under guidance of both techniques. Non-radical TUR cases based on the surgeon's intra-operative impression, patients without evidence of urothelial carcinoma in the pathology specimen, as well as those with isolated carcinoma in situ or muscle invasive disease were excluded from the study.

To generate the WL control cohort, data of all patients submitted to WL-TUR or biopsies for bladder cancer in our centre from January 2000 to September 2007 were retrospectively evaluated. Eligible patients were filtered out using the aforementioned exclusion criteria and were scored based on the matching criteria defined above. Finally, a random subset of patients was included: for each matching group triple the number of NBI-patients in that group, using nQuery Advisor 7.0.

All TURs performed in our centre (WL and NBI cohort) are standardised: for all tumours a deep biopsy is taken and sent to the pathologist in a separate container. Random cold cup biopsies are only taken in case of positive (high grade) urine cytology and negative cystoscopy. The quality of the video systems used for both cohorts was comparable: NBITUR was performed with $30^{\circ}$ optics connected to an Olympus Evis Exera II xenon light source and WL-TUR was performed with Hopkins II $30^{\circ}$ optics connected to a Karl Storz Xenon 300 light source.

Details of the selection process are presented in Fig. 1.

\section{Sample size analysis}

Based on prior studies on residual tumour rate at re-TUR following photodynamic diagnosis (PDD)-assisted TUR [11] we considered an absolute difference of $20 \%$ in RR-fFU between NBI-TUR and WL-TUR as clinically relevant. Taking into account that non-radical TURs were to be excluded from this study, we estimated an RR-fFU of $30 \%$ for the WL-TUR and an RR-fFU of $10 \%$ for the NBI-TUR cohort. Using a one-sided continuity corrected two sample Chi-square test and setting the probability of type I error $(\alpha)$ at 0.05 and type II error $(\beta)$ at 0.20 (desired power $80 \%$ ), the sample sizes of the index and the control groups with a ratio 1:3 were calculated to be 40 and 120 for NBI and WL, respectively (total sample size $n=160$ ). We chose for a $1: 3$ ratio in order to increase the power of the study. We opted for a one-sided test, instead of the more common two-sided approach, because NBI is intended to be used as an add-on to WL, rather than a replacement. In this study, NBI-TUR indeed consisted of resection under guidance of both WL and $\mathrm{NBI}$, and therefore, this procedure can only result in either equal or improved residual tumour rates, i.e. the alternative hypothesis has only one direction [12]. Sample size calculation was performed with the use of nQuery Advisor 7.0. 
Fig. 1 Flow chart of patient selection. TUR transurethral resection, NBI Narrow band imaging, $P D D$ Photodynamic diagnosis, CIS Carcinoma in situ, $U C$ Urothelial carcinoma, $f F U$ first follow-up

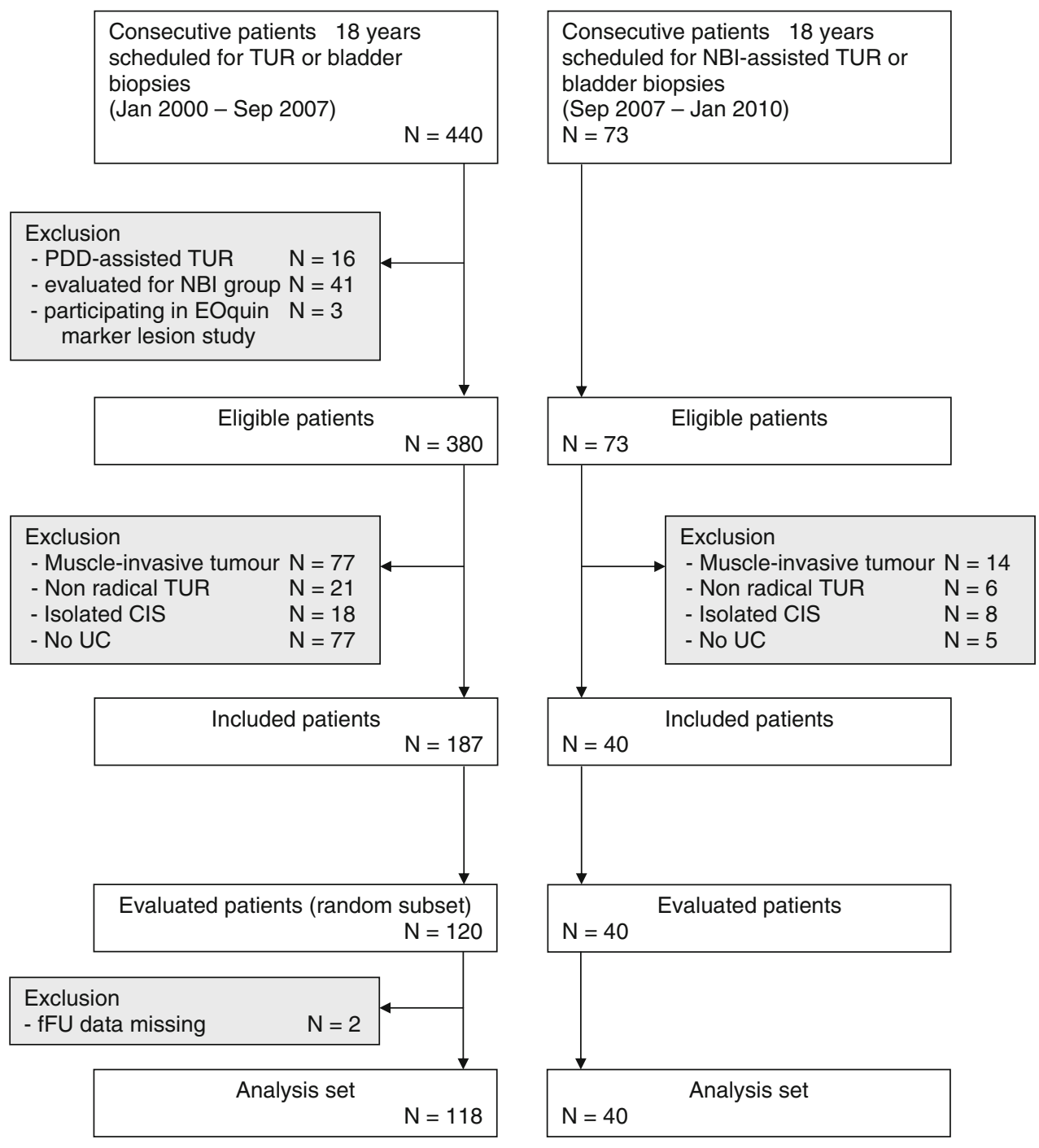

Outcome measures and analysis

For each patient the results of the fFU were recorded. If this consisted of cystoscopy plus cytology 3 months after the TUR, written reports and recorded still images of suspicious lesions were available. If a second TUR was performed to confirm pathological stage after the initial TUR and before the fFU cystoscopy at 3 months, the results of this procedure were used as fFU data. Since virtually all tumours identified at re-TUR and most tumours identified at 3-month cystoscopy in fact are residual/overlooked tumours rather than true recurrences, we present the findings of fFU as residual tumours.

Since frequency-matching design does not require a matched data analysis [13], baseline categorical variables as well as the main variable of interest (residual tumour at fFU in the two cohorts) and baseline continuous variables were analysed using Chi-square test, Fisher-exact test and independent samples $t$ test, respectively. Complementary, unconditional logistic regression analysis was performed to test the effect of intervention type (WL- or NBI-TUR) on the residual tumour rate at fFU, after adjusting for the matching variable to avoid odds ratio (OR) and its onesided $95 \%$ confidence interval $(\mathrm{CI})$ being biased by the procedure of frequency matching [13].

Statistical analysis was performed using the Statistical Package for Social Sciences, version 16.0 (SPSS, Chicago, Ill., USA) and $P<0.05$ was considered statistically significant.

\section{Results}

All baseline characteristics were comparable between groups (Table 1).

For the WL cohort, the fFU data were obtained by cystoscopy and cytology in 97 patients $(80.8 \%)$, by re-TUR in $17(15.9 \%)$ and in two $(1.7 \%)$ patients a cystectomy was 
Table 1 Baseline characteristics
${ }^{a}$ independent $t$ test

* Chi-square

NBI Narrow band imaging, $W L$ white light, CIS Carcinoma in situ, $M M C$ Mitomycin C, $B C G$ Bacillus Calmette-Guérin, $K L H$ keyhole limpet haemocyanin

\begin{tabular}{|c|c|c|c|c|}
\hline & NBI & WL & $\chi^{2}(d f)$ or $\mathrm{F}(d f)$ & $P$ value \\
\hline Number of patients & 40 & 120 & - & - \\
\hline \multicolumn{5}{|l|}{$\operatorname{Sex}(\%)$} \\
\hline Male & $30(75.0)$ & $86(71.7)$ & $0.17(1)$ & $0.68 *$ \\
\hline Female & $10(25.0)$ & $34(28.3)$ & & \\
\hline Age, mean (SD) yrs & $67.9(13.2)$ & $67.8(9.6)$ & $8.62(158)$ & $0.94^{\mathrm{a}}$ \\
\hline \multicolumn{5}{|l|}{ Number of tumours $(\%)$} \\
\hline Unifocal & $19(47.5)$ & $66(55.0)$ & $0.68(1)$ & $0.41 *$ \\
\hline Multifocal & $21(52.5)$ & $54(45.0)$ & & \\
\hline \multicolumn{5}{|l|}{ Prior recurrence rate $(\%)$} \\
\hline Primary & $28(70.0)$ & $70(58.3)$ & $1.72(1)$ & $0.19 *$ \\
\hline Recurrence & $12(30.0)$ & $50(41.7)$ & & \\
\hline \multicolumn{5}{|l|}{ Stage $(\%)$} \\
\hline $\mathrm{Ta}$ & $28(70.0)$ & $91(75.8)$ & $0.54(1)$ & $0.46^{*}$ \\
\hline $\mathrm{T} 1$ & $12(30.0)$ & $29(24.2)$ & & \\
\hline \multicolumn{5}{|l|}{ Grade $(\%)$} \\
\hline 1 & $3(7.5)$ & $11(9.2)$ & $2.45(2)$ & $0.29 *$ \\
\hline 2 & $15(37.5)$ & $60(50.0)$ & & \\
\hline 3 & $22(55.0)$ & $49(40.8)$ & & \\
\hline Concomitant CIS (\%) & $4(10.0)$ & $9(7.5)$ & $0.25(1)$ & $0.62 *$ \\
\hline \multicolumn{5}{|l|}{ EORTC risk group (\%) } \\
\hline Low & $2(5.0)$ & $6(5.0)$ & $0.00(2)$ & $1.00 *$ \\
\hline Intermediate & $33(82.5)$ & $99(82.5)$ & & \\
\hline High & $5(12.5)$ & $15(12.5)$ & & \\
\hline Immediate instillation (\%) & $17(42.5)$ & $51(42.5)$ & 0.00 & $1.00 *$ \\
\hline \multicolumn{5}{|l|}{ Adjuvant instillations (\%) } \\
\hline None & $23(57.5)$ & $69(57.5)$ & $0.32(2)$ & $0.85^{*}$ \\
\hline Chemotherapy & $6(15.0)$ & $22(18.3)$ & & \\
\hline MMC & $2(5.0)$ & $11(9.2)$ & & \\
\hline MMC + hyperthermia & $4(10.0)$ & $2(1.7)$ & & \\
\hline EOquin & $0(0.0)$ & $3(2.5)$ & & \\
\hline KLH & $0(0.0)$ & $5(4.2)$ & & \\
\hline Epirubicin & $0(0.0)$ & $1(0.8)$ & & \\
\hline Immunotherapy (BCG) & $11(27.5)$ & $29(24.2)$ & & \\
\hline
\end{tabular}

one-sided 95\% CI 1.2-6.1, $P=0.03$ ). Pathology results of the recurrences are displayed in Table 2.

\section{Discussion}

This is the first report on the impact of NBI-TUR on residual tumour rate. It has been previously shown that NBI cystoscopy significantly improves NMIBC detection since additional tumours missed during WL cystoscopy were seen using NBI technology, in $22-56 \%$ of the patients [6, 14-17]. Consequently, it is expected that NBI improves TUR quality by contributing to resection completeness, thereby reducing the residual tumour rate. In the current study we provide evidence to support this concept, showing 
Table 2 Pathology results of residual tumours
* Fisher-exact test

NBI Narrow band imaging, $W L$ white light

\begin{tabular}{|c|c|c|c|}
\hline & NBI & WL & $P$ value \\
\hline Patients with residual tumour (\%) & $6(15.0)$ & $36(30.5)$ & $0.04 *$ \\
\hline \multicolumn{4}{|l|}{ Stage $(\%)$} \\
\hline $\mathrm{Ta}$ & $3(50.0)$ & $19(52.8)$ & - \\
\hline $\mathrm{T} 1$ & $2(33.3)$ & $5(13.9)$ & \\
\hline $\mathrm{T} 2$ & $1(16.7)$ & $1(2.8)$ & \\
\hline Tis only & $0(0.0)$ & $4(11.1)$ & \\
\hline Unknown & $0(0.0)$ & $7(19.4)$ & \\
\hline Laser treatment & - & 4 & \\
\hline No pathology obtained because of comorbidity & - & 2 & \\
\hline Biopsy of insufficient quality for staging & - & 1 & \\
\hline \multicolumn{4}{|l|}{ Grade $(\%)$} \\
\hline 1 & $1(16.7)$ & $2(5.6)$ & - \\
\hline 2 & $0(0.0)$ & $13(36.1)$ & \\
\hline 3 & $4(66.7)$ & $15(41.7)$ & \\
\hline Unknown & $1(16.7)$ & $6(16.7)$ & \\
\hline Laser treatment & - & 4 & \\
\hline No pathology obtained because of comorbidity & - & 2 & \\
\hline Biopsy of insufficient quality for grading & 1 & - & \\
\hline Residual tumour upstaged/upgraded if compared to initial tumour(s) & $2(33.3)$ & $6(16.7)$ & - \\
\hline
\end{tabular}

that the residual tumour rate after NBI-TUR is significantly lower compared to standard WL-TUR (15 vs. 30.5\%, respectively). Based on the logistic regression analysis, we can state that the odds for detecting a tumour up to 3 months after WL-TUR is 2.7 times higher than after NBI-TUR.

Potential limitations of our study are related to its design, namely the retrospective collection of the control group. First, it may be speculated that retrospectively collected data including baseline characteristics or information on TUR radicality was more difficult to retrieve. Nevertheless, there were no missing data on baseline characteristics, which underlines the documentation quality. In only two patients $(1.7 \%)$ data on fFU was lacking, due to non-specific circumstances. For all other WL-cohort patients, results of fFU cystoscopy were well documented with an electronic report supplemented by bladder diagram, recorded still images of abnormal looking urothelium and bladder-wash out cytology. In cases with re-TUR, the surgical as well as the final pathological report were available electronically for all patients. Second, the WL-TUR cohort was treated in an earlier era than the NBI-patients, which may have influenced the outcome. However, the treatment strategy has not been altered over these years: the first EAU guidelines on bladder cancer already advocated a complete TUR, supplemented by the administration of one single chemotherapeutic intravesical instillation immediately after TUR, and adjuvant instillations in the intermediate- and high risk group [18]. This is illustrated by the similar number of patients with adjuvant courses of intravesical therapy. In addition, the type of adjuvant therapy was not significantly different for the two intervention groups (Table 1). Third, despite the fact that re-TUR is the best proof of absence of residual tumour, a re-TUR was not incorporated as a standard procedure in the study protocol due to the partly retrospective design of the study. Thus, most fFU data were based on outpatient flexible cystoscopy findings. Nevertheless, almost $33 \%$ of patients in the NBI group and $16 \%$ in the WL group had a re-TUR as part of routine management. It has to be noted that in all these patients, re-TUR was performed to ensure correct staging rather than due to doubt on radicality of the first TUR (which was an exclusion criterion). The difference in re-TUR ratios between the two groups may be explained by the relatively higher number of pT1 and/or grade 3 tumours in the NBI group (though not statistically significant), necessitating confirmation of non-muscle invasive disease.

To compare the effect of two interventions, it is crucial that groups are identical on potential confounders. In this study, we generated two comparable intervention groups by frequency matching based on the EORTC risk criteria and the single intravesical chemotherapy instillation status immediately after TUR. These two factors are considered the most important determinants of future bladder cancer recurrence [19, 20]. 
Attempting to decrease the well-known high number of early recurrences in NMIBC is of utmost importance because these recurrences necessitate meticulous followup with cystoscopy plus cytology and adjuvant courses of intravesical therapy in selected cases, all of which result in high patients' burden and increased costs. In fact, it has been estimated that bladder cancer is one of the most expensive cancers of today's medical practice, mainly due to the lifelong need for follow-up and repeated treatments [21]. Many studies have already proven that PDD is effective in reducing the early recurrence rate [22-25]. However, there are some drawbacks of PDD that have restricted a widespread use. Since NBI does not require an intravesical fluorescent it has advantages over PDD, provided that the system proves to be equally effective. Theoretically, the expenditures probably will be lower, there is no risk of side-effects and the technique is immediately applicable without necessitating a special patient preparation.

Of course additional randomized and/or multicentre trials are warranted to confirm these first promising results. However, despite the lower level of evidence that the current study provides, it is important to have this evidence before investing time and money in constructing a randomised controlled trial. Partly triggered by the results of this trial, the clinical research office of the endourological society is currently doing a multicentre, international, randomised trial on the effect of NBI-assisted TUR on recurrence rates [26]. In addition, future studies have to investigate whether this improved outcome at 3 months will sustain at longer follow-up. Another interesting objective for future research could be to compare the effect of standard WL-TUR followed by adjuvant intravesical therapy with NBI-TUR without this adjuvant therapy. If the NBI-TUR can influence recurrence rate to such an extent that these intravesical therapies become redundant, this would have a major impact on healthcare, both for the patients (increased quality of life) as well as the economics (less healthcare costs).

\section{Conclusions}

This is the first study demonstrating that NBI-TUR for NMIBC decreases residual tumour rate significantly when compared to a matched cohort of WL-TUR.

Residual tumour rate was $30.5 \%$ in patients treated by WL-TUR and $15.0 \%$ in patients treated by NBI-TUR, thus a statistically significant absolute difference of $15.5 \%$ in favour of NBI-TUR.

Acknowledgments We acknowledge Olympus Europa Holding $\mathrm{GmbH}$, Hamburg, Germany, for providing the NBI system.
In addition, we would like to thank N. van Geloven and Professor A.H. Zwinderman from the Department of Clinical Epidemiology and Biostatistics (Academic Medical Center, Amsterdam) for their assistance in statistical analysis.

Conflict of interest The authors declare that they have no conflict of interest.

Open Access This article is distributed under the terms of the Creative Commons Attribution Noncommercial License which permits any noncommercial use, distribution, and reproduction in any medium, provided the original author(s) and source are credited.

\section{References}

1. Brausi M, Collette L, Kurth K, van der Meijden AP, Oosterlinck W, Witjes JA, Newling D, Bouffioux C, Sylvester RJ (2002) Variability in the recurrence rate at first follow-up cystoscopy after TUR in stage Ta T1 transitional cell carcinoma of the bladder: a combined analysis of seven EORTC studies. Eur Urol 41:523-531

2. Han KS, Joung JY, Cho KS, Seo HK, Chung J, Park WS, Lee KH (2008) Results of repeated transurethral resection for a second opinion in patients referred for nonmuscle invasive bladder cancer: the referral cancer center experience and review of the literature. J Endourol 22:2699-2704

3. Herr HW (2005) Surgical factors in the treatment of superficial and invasive bladder cancer. Urol Clin North Am 32:157-164

4. Schwaibold HE, Sivalingam S, May F, Hartung R (2006) The value of a second transurethral resection for T1 bladder cancer. BJU Int 97:1199-1201

5. Dwivedi US, Kumar A, Das SK, Trivedi S, Kumar M, Sunder S, Singh PB (2009) Relook TURBT in superficial bladder cancer: its importance and its correlation with the tumor ploidy. Urol Oncol 27:514-519

6. Cauberg EC, Kloen S, Visser M, de la Rosette JJ, Babjuk M, Soukup V, Pesl M, Duskova J, de Reijke TM (2010) Narrow Band Imaging Cystoscopy Improves the Detection of Non-muscle-invasive Bladder Cancer. Urology 76:658-663

7. Sylvester RJ, van der Meijden AP, Oosterlinck W, Witjes JA, Bouffioux C, Denis L, Newling DW, Kurth K (2006) Predicting recurrence and progression in individual patients with stage $\mathrm{Ta}$ T1 bladder cancer using EORTC risk tables: a combined analysis of 2596 patients from seven EORTC trials. Eur Urol 49:466-475

8. Babjuk M, Oosterlinck W, Sylvester R, Kaasinen E, Bohle A, Palou-Redorta J (2008) EAU Guidelines on Non-Muscle-Invasive Urothelial Carcinoma of the Bladder. Eur Urol 54:303-314

9. Sobin LH, Wittekind Ch (2002) TNM classification of malignant tumours, 6th edn. pp 199-202

10. Mostofi FK, Sobin LH, Torloni H (1973) Histological typing of urinary bladder tumours. International classification of tumors, 19th World Health Organization: Genova, Switzerland,pp 17

11. Kausch I, Sommerauer M, Montorsi F, Stenzl A, Jacqmin D, Jichlinski P, Jocham D, Ziegler A, Vonthein R (2010) Photodynamic Diagnosis in Non-Muscle-Invasive Bladder Cancer: A Systematic Review and Cumulative Analysis of Prospective Studies. Eur Urol 57:595-606

12. Knottnerus JA, Bouter LM (2001) The ethics of sample size: two-sided testing and one-sided thinking. J Clin Epidemiol 54:109-110

13. Cheung YB (2003) Analysis of matched case-control data. J Clin Epidemiol 56:814

14. Bryan RT, Billingham LJ, Wallace DM (2008) Narrow-band imaging flexible cystoscopy in the detection of recurrent urothelial cancer of the bladder. BJU Int 101:702-705 
15. Herr HW, Donat SM (2008) A comparison of white-light cystoscopy and narrow-band imaging cystoscopy to detect bladder tumour recurrences. BJU Int 102:1111-1114

16. Herr HW (2010) Narrow-band imaging cystoscopy to evaluate the response to bacille Calmette-Guerin therapy: preliminary results. BJU Int 105:314-316

17. Naselli A, Introini C, Bertolotto F, Spina B, Puppo P (2010) Narrow band imaging for detecting residual/recurrent cancerous tissue during second transurethral resection of newly diagnosed nonmuscle-invasive high-grade bladder cancer. BJU Int 105:208-211

18. Oosterlinck W, Lobel B, Jakse G, Malmstrom PU, Stockle M, Sternberg C (2002) Guidelines on bladder cancer. Eur Urol 41:105-112

19. Sylvester RJ, Oosterlinck W, van der Meijden AP (2004) A single immediate postoperative instillation of chemotherapy decreases the risk of recurrence in patients with stage Ta $\mathrm{T} 1$ bladder cancer: a meta-analysis of published results of randomized clinical trials. J Urol 171:2186-2190

20. van Rhijn BW, Burger M, Lotan Y, Solsona E, Stief CG, Sylvester RJ, Witjes JA, Zlotta AR (2009) Recurrence and progression of disease in non-muscle-invasive bladder cancer: from epidemiology to treatment strategy. Eur Urol 56:430-442

21. Botteman MF, Pashos CL, Redaelli A, Laskin B, Hauser R (2003)

The health economics of bladder cancer: a comprehensive review of the published literature. Pharmacoeconomics 21:1315-1330
22. Daniltchenko DI, Riedl CR, Sachs MD, Koenig F, Daha KL, Pflueger H, Loening SA, Schnorr D (2005) Long-term benefit of 5-aminolevulinic acid fluorescence assisted transurethral resection of superficial bladder cancer: 5-year results of a prospective randomized study. J Urol 174:2129-2133

23. Denzinger S, Burger M, Walter B, Knuechel R, Roessler W, Wieland WF, Filbeck T (2007) Clinically relevant reduction in risk of recurrence of superficial bladder cancer using 5-aminolevulinic acid-induced fluorescence diagnosis: 8-year results of prospective randomized study. Urology 69:675-679

24. Denzinger S, Wieland WF, Otto W, Filbeck T, Knuechel R, Burger M (2008) Does photodynamic transurethral resection of bladder tumour improve the outcome of initial T1 high-grade bladder cancer? A long-term follow-up of a randomized study. BJU Int 101:566-569

25. Kriegmair M, Zaak D, Rothenberger KH, Rassweiler J, Jocham D, Eisenberger F, Tauber R, Stenzl A, Hofstetter A (2002) Transurethral resection for bladder cancer using 5-aminolevulinic acid induced fluorescence endoscopy versus white light endoscopy. J Urol 168:475-478

26. de la Rosette J, Gravas S (2010) A multi-center, randomized international study to compare the impact of narrow band imaging versus white light cystoscopy in the recurrence of bladder cancer. J Endourol 24:660-661 